Statistical Analysis and Time Series Models for Minimum/Maximum Temperatures in the Antarctic Peninsula

Hughes, Gillian and Subba Rao, T

2006

MIMS EPrint: 2006.36

Manchester Institute for Mathematical Sciences

School of Mathematics

The University of Manchester

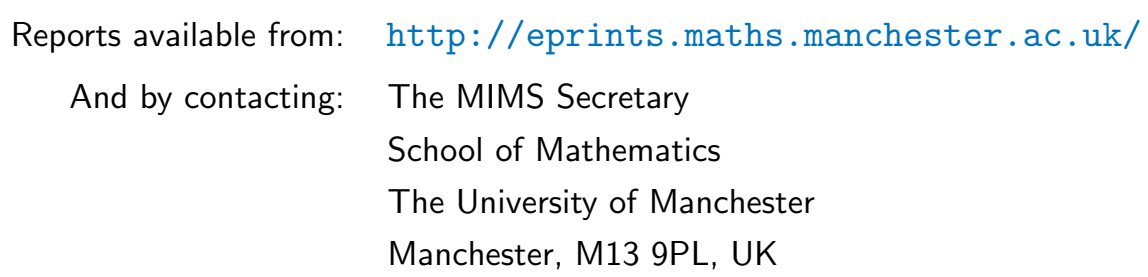

ISSN 1749-9097 
Statistical Analysis and Time Series Models for Minimum/Maximum Temperatures in the Antarctic Peninsula

Gillian L. Hughes \& Tata Subba Rao

First version: 23 September 2005

Research Report No. 5, 2005, Probability and Statistics Group School of Mathematics, The University of Manchester 


\title{
Statistical analysis and time series models for minimum/maximum temperatures in the Antarctic Peninsula
}

\author{
By Gillian L. Hughes and Tata Subba Rao \\ University of Manchester, \\ School of Mathematics, \\ Manchester M60 1QD, \\ $U . K$.
}

\begin{abstract}
Our object in this paper is to study the temperature variations in the Antarctic Peninsula using multiple regression models with correlated errors admitting ARMA models with nonGaussian innovations. We found that the fitted models adequately describe the variations. The data we consider are minimum/maximum monthly temperatures recorded at the Faraday station by the British Antarctic Survey for the period from January 1951 to December 1995. The time series models considered here are novel in the sense that the linear ARMA models have innovations which have extreme value distributions, and the maximum likelihood estimation described here can be widely used in many disciplines.

The time series models we fitted indicate that the mean of the minimum temperatures is likely to increase over the next 50 years and the temperatures will be above $0^{\circ} \mathrm{C}$ during the summer months which means that the melting season will increase, creating more climatic and ecological problems. Although the mean temperature is reported to have increased by $2.5^{\circ} \mathrm{C}$ we believe that the maximum temperatures have remained unchanged over the past 45 years. This has led to a decrease in the diurnal temperature range which has also been observed in many other parts of the globe.

The influence of human activity on climate is still unknown but our ability to perturb the ozone layer is an established fact. We established a relationship between minimum monthly temperatures and ozone levels and found they are highly negatively correlated (at a lag of one month) implying that the higher levels of ozone in the air keep temperatures low. This resulted in a new time series model relating the minimum temperatures to ozone levels. After appropriate statistical tests, we have come to the conclusion that the observed increase in the minimum temperatures is a consequence of human activity rather than natural causes and so a reduction in the production of "greenhouse gases" could lead to a decrease in minimum temperatures, thereby reducing the adverse effect of global warming in the Antarctic Peninsula.
\end{abstract}

Keywords: Antarctic pensinsula, ozone levels, temperatures, time series models 


\section{Introduction}

The data considered here are minimum monthly temperatures from the Faraday research station on the Antarctic Peninsula from January 1951 to December 1995. The data set consists of 540 observations.

This particular station is of interest because it has the longest continuous occupation of any British station to date. It has been observed that, over the past 50 years, the annual mean temperatures at this station have risen by approximately $2.5^{\circ} \mathrm{C}$ (British Antarctic Survey (BASa), (2002)) and the small fringing ice shelves around the Antarctic Peninsula are retreating (Hulbe, (1997)). The change in local climate is also demonstrated by profound ecological changes.

Ice shelves are platforms of floating ice which are hundreds of meters thick. Today they comprise about $2 \%$ of Antarctic ice and they form where inland glaciers and ice sheets discharge into the ocean. Mass is lost primarily by iceberg calving at the seaward ice cliff and secondarily by melting at the lower surface. Changes in local climate affect ice shelf mass balance, that is, the difference between the mass of ice gained and lost by the shelf, and therefore the size of the shelf and the location of its calving front.

An increase in atmospheric temperature, if large enough to push summer temperatures above freezing point, will increase mass loss directly by increasing melting at the upper surface. Warmer sea surface temperatures (SST) may accompany warmer air temperatures and this could also increase the rate of ice shelf melting. There are also indirect effects of warmer air temperatures that can hasten the decay of ice shelves.

The effects of $\mathrm{CO}_{2}$-induced climate warming on Antarctica have been studied using numerical models that simulate ice flow and changes in ice sheet and ice shelf size over time (Huybrechts and Oerlemans, (1990) and Budd et al, (1994)). One prediction of the models is that the glaciers and ice shelves of the Peninsula are lost.

Although the mean air temperature is reported to have increased by $2.5^{\circ} \mathrm{C}$, we believe that it may be the minimum temperatures which have increased rather than the maximum temperatures and our analysis in this paper supports this belief.

An increase in minimum temperatures could have a more dramatic effect on the surroundings than an overall increase in temperatures. Clearly if the minimum temperatures rise so that they are consistently above freezing point then the ice shelves will lose mass directly, and indirectly, very quickly. The length of time surface ice melts, or the 'melt season', has been shown to be particularly important in the break-up of ice shelves.

There is clearly a connection between warming around the Antarctic Peninsula and the collapse of Peninsula ice shelves and, unless there is a change in the observed warming trend, further retreats of fringing of ice shelves along the Antarctic Peninsula are inevitable. It is therefore important to forecast how quickly the temperatures are going to increase above melting point as this is vital to the break-up of ice shelves. Clearly if the minimum summer temperatures increase above $0^{\circ} \mathrm{C}$ the melt season will be tremendously long and the ice shelves are likely to become very unstable. Our study attempts to analyse the trend in the minimum monthly temperatures and forecast when these minima are expected to be greater than the melting point in the summer months. We consider both maximum monthly tem- 


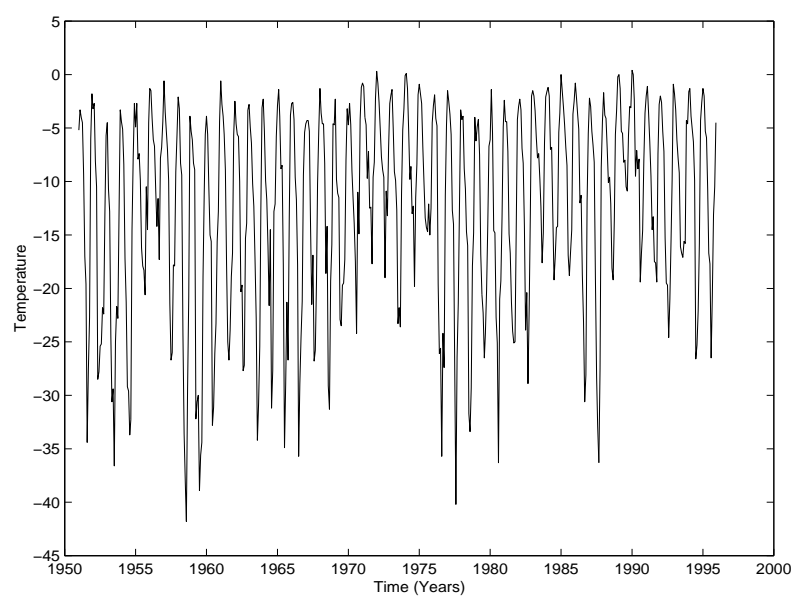

Figure 1. Minimum monthly temperatures at the Faraday station (1951-1995)

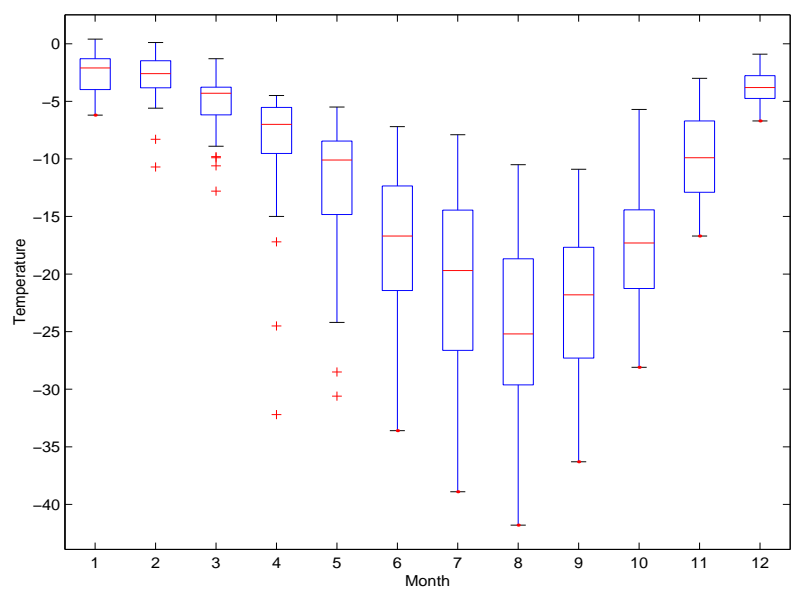

Figure 2. Boxplot by month of minimum monthly temperatures

peratures and minimum monthly temperatures, though our main emphasis is on minimum temperatures.

\section{Data analysis of Minimum Monthly Temperatures}

In figure 1, the minimum monthly temperature series from January 1951 to December 1995 is plotted and we see the winter temperatures have been increasing rapidly while the summer temperatures have been increasing more slowly or remaining constant, consequently it appears that the range of the series has been decreasing over time. The increasing trend appears to be linear.

The boxplots of the minimum monthly temperature data by month where month 1 is January, month 2 is February and so on is given in figure 2. Clearly there is a yearly cycle but it is also seen that there is a much larger spread of temperatures 


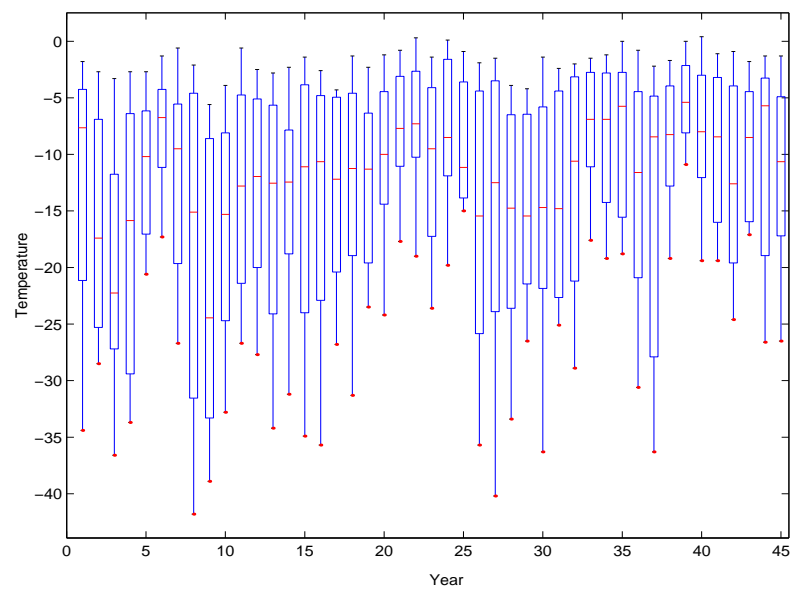

Figure 3. Boxplot by year of minimum monthly temperatures

in the winter months, that is, from June to August. There is some indication that the variance is changing with the season. During the winter months there are no 'extreme values' lying outside of the 'whisker' on the boxplots. The whisker shown is 1.5 times the inter-quartile range. Extreme values only exist for February to May and these are only on the lower temperature side, implying that the extreme temperatures are lower and not higher than the median. The boxplot of the minimum monthly temperatures from the Faraday station by year where year 1 is 1951 up to year 45 which is 1995 is given in figure 3 . There are two big decreases in median values in years 3 and 9 corresponding to years 1953 and 1959. The upper whisker of the boxplots are reasonably level but the lower whiskers fluctuate by a considerable amount, with the amount of variation in temperatures decreasing over the years. The median of the boxplots also seem to have increased over time.

\section{Data analysis of Maximum Monthly Temperatures}

We now analyse the maximum monthly temperatures to see whether there is any asymmetric climate warming of the Faraday Station. A plot of the maximum monthly temperature data is given in figure 4 . The box plots by month and by year of this data are given in figures 5 and 6 respectively.

The range of this series, $\left(-8^{\circ} \mathrm{C}, 10^{\circ} \mathrm{C}\right)$, is quite small compared to the minimum monthly temperatures. There is not a significant change over time compared to the minimum temperatures. From the box plot given in figure 5, we see that there is possibly a yearly cycle and in most months some extreme values have occurred. Figure 6 shows that there does not appear to be any significant changes over the 45 year period. The median and range of values over the 45 years seems to have remained quite constant.

From the above preliminary data analysis, we see that minimum temperatures are exhibiting more dynamic behaviour. In the following we briefly discuss the estimation of multiple regression models where the errors are correlated and the innovations have extreme value distributions. 


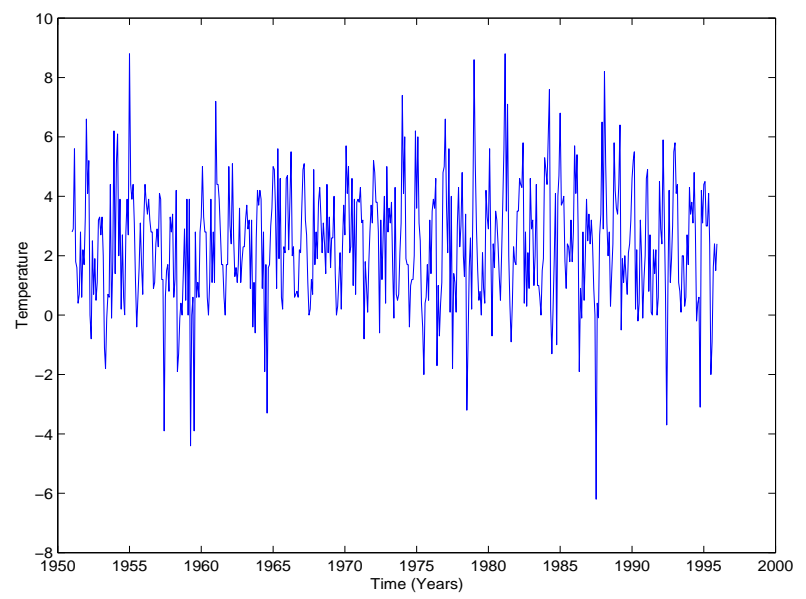

Figure 4. Maximum monthly temperatures at the Faraday station (1951-1995)

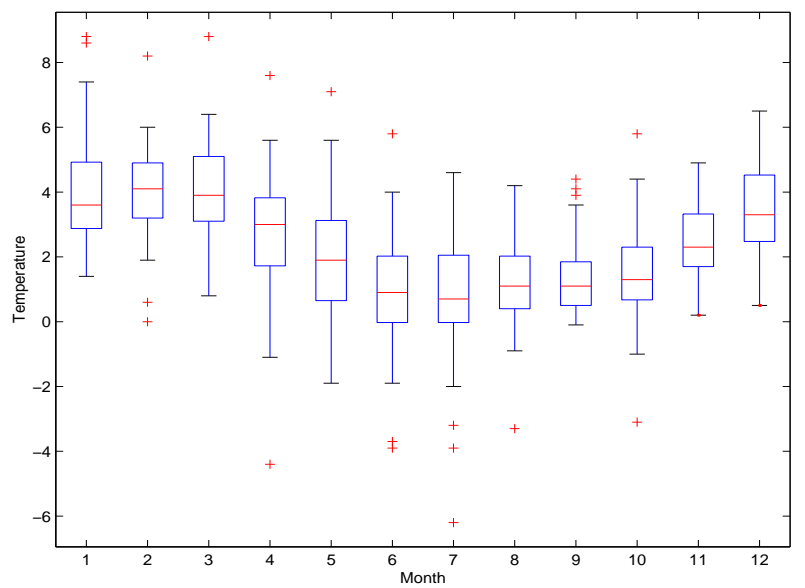

Figure 5. Boxplot by month of maximum monthly temperatures

\section{Multiple Regression with Correlated Errors}

In this section we consider the maximum likelihood estimation of the parameters of a classical multiple regression model with correlated errors, when the errors satisfy an Autoregressive Moving Average (ARMA) model with innovations having a Generalised Extreme Value (GEV) distribution (see appendix A). Suppose we observe the time series $\left\{y_{t}\right\}$, where $y_{t}$ is given by

$$
y_{t}=\sum_{j=0}^{r} \beta_{j} x_{j, t}+e_{t} ; t=1, \ldots, n
$$




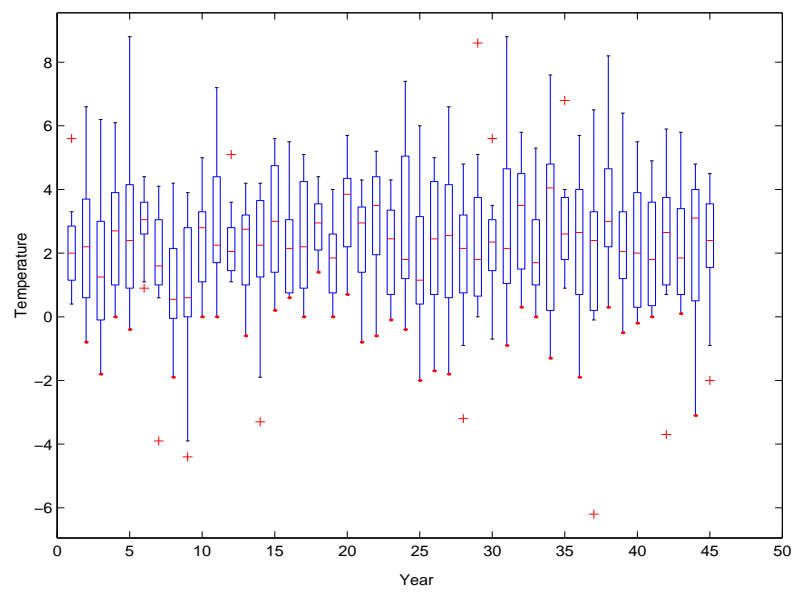

Figure 6 . Boxplot by year of maximum yearly temperatures

where $\left\{e_{t}\right\}$ satisfy the stationary $A R M A(p, q)$ model

$$
\begin{aligned}
\left(1+\phi_{1} B+\phi_{2} B^{2}+\cdots+\phi_{p} B^{p}\right) e_{t} & =\left(\theta_{1} B+\theta_{2} B^{2}+\cdots+\theta_{q} B^{q}\right) \eta_{t} \\
\text { or equivalently } \phi_{p}(B) e_{t} & =\theta_{q}(B) \eta_{t}
\end{aligned}
$$

where $\left\{\eta_{t}\right\}$ are independent, identically distributed random variables where each $\eta_{t}$ has the converse $\operatorname{GEV}(\gamma, \mu, \sigma)$ distribution. We assume the regressor variables $\left\{x_{j t}\right\}$ are non random and the time series $\left\{e_{t}\right\}$ generated by (4.2) is stationary and the model is invertible. Using (4.2) we can write (4.1) as

$$
y_{t}=\sum_{j=0}^{r} \beta_{j} x_{j, t}+\phi_{p}(B)^{-1} \theta_{q}(B) \eta_{t}
$$

or, equivalently,

$$
\eta_{t}=\theta_{q}(B)^{-1} \phi_{p}(B)\left[y_{t}-\sum_{j=0}^{r} \beta_{j} x_{j, t}\right] ; \quad(t=1,2, \ldots, n) .
$$

Let $s=\max (p, q)$. Assuming the probability density function (pdf) of $\eta_{t}$ is given by

$$
\begin{aligned}
f_{\eta}(y)= & \frac{1}{\sigma} \exp \left\{-\left[1+\gamma\left(\frac{-y+\mu_{Y}}{\sigma}\right)\right]^{-\frac{1}{\gamma}}\right\}\left[1+\gamma\left(\frac{-y+\mu_{Y}}{\sigma}\right)\right]^{-\left(1+\frac{1}{\gamma}\right)} ; \\
& \text { for } 1+\gamma\left(\frac{-y+\mu_{Y}}{\sigma}\right)>0
\end{aligned}
$$

we can write the conditional log-likelihood function of $\left(\eta_{s+1}, \eta_{s+2}, \ldots, \eta_{n}\right)$ as

$$
\begin{aligned}
l(\eta ; \underline{\beta}, \underline{\phi}, \underline{\theta}, \gamma, \mu, \sigma)= & -(n-s) \log \sigma-\left(\frac{1}{\gamma}+1\right) \sum_{t=s+1}^{n} \log \left[1+\gamma\left(\frac{\mu-\eta_{t}}{\sigma}\right)\right] \\
& -\sum_{t=s+1}^{n}\left[1+\gamma\left(\frac{\mu-\eta_{t}}{\sigma}\right)\right]^{-\frac{1}{\gamma}}
\end{aligned}
$$


provided $1+\gamma\left(\frac{\mu-\eta_{t}}{\sigma}\right)>0$ for each $t$, where $\underline{\beta}=\left(\beta_{0}, \beta_{1}, \ldots, \beta_{s}\right), \underline{\phi}=\left(\phi_{1}, \phi_{2}, \ldots, \phi_{p}\right)$, $\underline{\theta}=\left(\theta_{1}, \theta_{2}, \ldots, \theta_{p}\right)$. In situations where $E\left(e_{t}\right) \neq 0$ and $x_{j, t}=0$, we can set $\beta_{0}=0$ since the mean of the converse GEV distribution is non-zero. This avoids over parameterisation. We note $\eta_{t}$ in (4.6) is unobservable and the estimation of the parameters $\beta, \phi, \underline{\theta}, \gamma, \mu, \sigma$ can only be done using iterative techniques which requires the evaluation of first order and second order partial derivatives with respect to the above parameters. This in turn requires obtaining an initial set of estimates. We require the second order partial derivatives for the calculation of the Fishers Information matrix. In the following we describe the steps for obtaining the initial estimates of the parameters.

1. First estimate the regression parameters, $\underline{\beta}$, by the method of ordinary least squares (OLS). Let $\underline{\hat{\beta}}$ be such an estimate.

2. Obtain

$$
\hat{e}_{t}=y_{t}-\sum_{j=0}^{r} \hat{\beta}_{j} x_{j, t} ; \quad\left(t=1,2, \ldots, n ; \quad \beta_{0}=0\right) .
$$

Test the residuals $\left\{\hat{e}_{t}\right\}$ for zero correlation (see Brockwell and Davis, (1996)). If we reject the null hypothesis we fit an $\operatorname{ARMA}(p, q)$ model to the various $\left\{\hat{e}_{t}\right\}$ using the Hannan-Rissanen (1982) method (c.f. Brockwell and Davis, (1996)). The orders $p$ and $q$ are chosen using Bayes Information Criterion (BIC). Let $\hat{p}$ and $\hat{q}$ be the chosen orders.

3. Using (4.4) we obtain the estimated residuals

$$
\hat{\eta}_{t}=\hat{\theta}_{q}(B)^{-1} \hat{\phi}_{p}(B)\left[y_{t}-\sum_{j=0}^{r} \hat{\beta}_{j} x_{j, t}\right] ; \quad t \geq 1+\hat{p}+\hat{q} .
$$

The residuals, $\left\{\hat{\eta}_{t}\right\}$, are tested for Gaussianity using the standard skewness and kurtosis measures (see D'Agostino and Stephens, (1996)). If we reject the null hypothesis, we have to search for an appropriate distribution. Since we are considering in this paper the minimum and maximum temperatures a natural family of distributions are extreme value distributions. The parameters $\gamma, \mu$ and $\sigma$ of the converse GEV distribution are estimated (Prescott and Walden, 1980) from the residuals $\left\{\hat{\eta}_{t}\right\}$ using the computer program xtremes (see Reiss and Thomas, (1997)).

The estimates obtained from the above steps will now be used as initial estimates for our iterative procedure. If we denote the parameter values by $\underline{\theta}$, and $\underline{\theta}^{(m)}$ be the estimate of the parameter vector $\underline{\theta}$ at stage $m$, then the updated estimate is given by

$$
\underline{\theta}^{(m+1)}=\underline{\theta}^{(m)}+\left[-\frac{\partial^{2} l}{\partial \underline{\theta}^{(m)} \partial \underline{\theta}^{(m) \prime}}\right]^{-1} \frac{\partial l}{\partial \underline{\theta}^{(m)}},
$$

where $l$ is the log-likelihood function.

Let $I(\underline{\theta})=E\left[-\frac{\partial^{2} l}{\partial \underline{\theta} \partial \underline{\theta}^{\prime}}\right]$ be the Fisher's information matrix. Then for large $n$ an appropriate expression for the variance-covariance matrix of $\underline{\hat{\theta}}$ is given by 
$\operatorname{Var}(\underline{\hat{\theta}}) \approx I^{-1}(\underline{\theta})$. The elements of the information matrix for the parameters of the model (4.1) where the log-likelihood function is given by (4.6) are given in Appendix B, which we use to estimate the standard errors of the estimates of the parameters. We assume that for large $n$ that $\underline{\hat{\theta}}$ has a multivariate normal distribution.

\section{Time Series Model for the Minimum Temperatures}

From the earlier data analysis, we see that there is a linear trend, and periodicity in the data. Since the data is monthly, we expect the periodicity will be equal to 12 months.

The model we consider for the minimum monthly temperatures $\left\{y_{t}\right\}$ is

$$
y_{t}=\sum_{j=0}^{r} \beta_{j} t^{j}+\sum_{j=1}^{k}\left[A_{j} \cos \left(\omega_{j} t\right)+B_{j} \sin \left(\omega_{j} t\right)\right]+e_{t} ; \quad\left(\beta_{0}=0\right) ;
$$

where $\sum_{j=0}^{p} \phi_{j} e_{t-j}=\sum_{j=0}^{q} \theta_{j} \eta_{t-j} ; \quad \phi_{0}=\theta_{0}=1 ;$

$$
\eta_{t} \sim \operatorname{conv} \operatorname{GEV}(\gamma, \mu, \sigma) .
$$

After fitting an appropriate model as described before, we propose a test for testing the goodness of fit of the residuals.

If the frequencies $\left\{\omega_{j}\right\}$ of the model (5.1) are assumed to be known, then the model (5.1) becomes a particular case of (4.1); otherwise we have to estimate the frequencies $\left\{\omega_{j}\right\}$. We use the method proposed by Quinn and Fernandes (1991) for estimating the frequencies and the Kavalieris and Hannan (1994) algorithm for choosing the number of frequencies. We don't give details here. We briefly describe the algorithm for estimating the parameters, including $\left\{\omega_{j}\right\}$, in the following steps.

\section{(a) Estimation procedure}

1. Estimate $\left\{\beta_{j}\right\}$ by the method of ordinary least squares. Let $\left\{\hat{\beta}_{j}\right\}$ be the estimates.

2. Estimate the residuals, $\left\{\varsigma_{t}\right\}$ say,

$$
\hat{\varsigma}_{t}=y_{t}-\sum_{j=0}^{r} \hat{\beta}_{j} t^{j} .
$$

Estimate the frequencies, $\left\{\omega_{j}\right\}$, and the number of frequencies using the Quinn and Fernandes algorithm to the residuals. Then obtain the new residuals

$$
\hat{e}_{t}=y_{t}-\sum_{j=0}^{r} \hat{\beta}_{j} t^{j}-\sum_{j=1}^{k}\left[\hat{A}_{j} \cos \left(\hat{\omega}_{j} t\right)+\hat{B}_{j} \sin \left(\hat{\omega}_{j} t\right)\right] .
$$


3. Using the Hannan-Rissanen algorithm (1982) fit an $\operatorname{ARMA}(p, q)$ model to $\left\{\hat{e}_{t}\right\}$. Let

$$
\hat{\eta}_{t}=\hat{\theta}_{q}^{-1}(B) \hat{\phi}_{p}(B) \hat{e}_{t} .
$$

Test for the independence and Gaussianity of $\left\{\hat{\eta}_{t}\right\}$. If the assumption of Gaussianity is rejected,we assume that the converse GEV distribution is appropriate and proceed using the maximum likelihood method given in section 4 for estimating $\underline{\beta}, \underline{\phi}, \underline{\theta}, \gamma, \mu, \sigma$. Here we assume $\left\{A_{j}, B_{j}, \omega_{j}\right\}$ are known and that maximisation is done with respect to the rest of the parameters. The orders $p$ and $q$ are chosen using the BIC.

The variances of the final estimates are calculated using both the observed information matrix and the expected information matrix using the expressions given in Appendix B.

By the search procedure we described, we found that the series can be best described by a linear trend, one periodic component (with a 12 month cycle) and an AR(1) model for the errors, and the innovations described by a converse GEV distribution.

The final model obtained is

$$
\begin{gathered}
y_{t}=\begin{array}{c}
0.008388 t+10.621 \cos (0.52291 t) \\
(0.0027,0.0026) \\
+2.5115 \sin (0.52291 t)+e_{t} \\
e_{t}-\underset{(0.031,0.032)}{0.59171} e_{t-1}=\eta_{t}
\end{array} \\
\eta_{t} \sim \text { converse GEV }\left(\begin{array}{l}
-0.12415,-4.309, \\
(0.019,0.026)
\end{array}, \begin{array}{c}
3.7238 \\
(0.59,0.58)
\end{array}\right)
\end{gathered}
$$

The numbers in parentheses are standard errors of the estimates, the first calculated from the observed information matrix and the second from the expected information matrix. The observed information matrix is the Hessian of the negative log-likelihood, evaluated at the maximum of the likelihood. Its inverse provides a good approximation to the variance-covariance matrix of the estimators. The expected information matrix is the expected value of the above Hessian. The good agreement between these two standard errors suggests that the estimation method is working satisfactorily.

\section{(b) Goodness of fit test by Probability Plots}

A probability plot is a graphical technique for assessing whether or not a data set follows a given distribution. Our object here is to test for the goodness of fit of the residuals $\left\{\hat{\eta}_{t}\right\}$ to confirm that our assumption that they follow a converse GEV distribution is appropriate. Suppose the random variable $X$ has a converse GEV distribution with parameters $(\gamma, \mu, \sigma)$, then if

$$
p_{t}=P\left(X \leq x_{t}\right)=1-\exp \left\{-\left[1+\frac{\sigma}{\gamma}\left(\mu-x_{t}\right)\right]^{-\frac{1}{\gamma}}\right\}
$$

and

$$
\gamma \log \left[-\log \left(1-p_{t}\right)\right]=-\log \left[1+\frac{\sigma}{\gamma}\left(\mu-x_{t}\right)\right]
$$




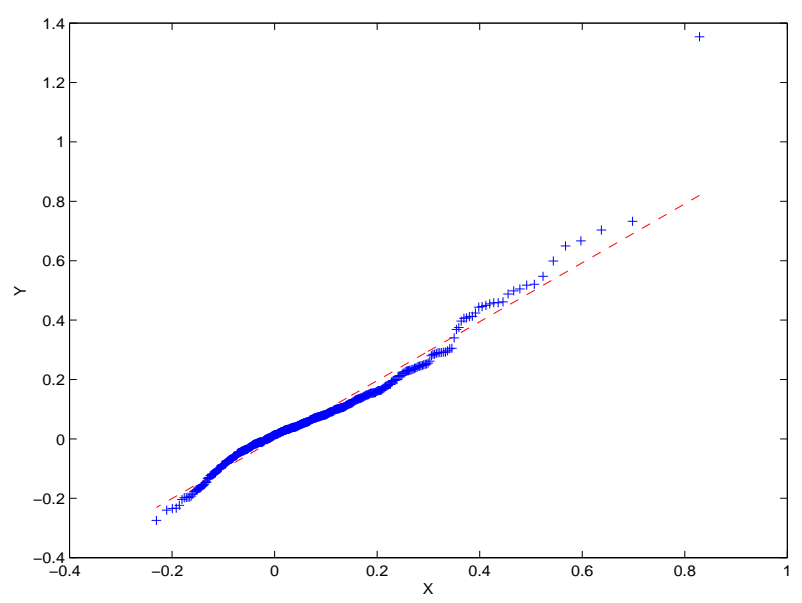

Figure 7. Validation plot

then the plot $\left\{-\log \left[1+\frac{\sigma}{\gamma}\left(\mu-x_{t}\right)\right]\right\}$ against $\gamma \log \left[-\log \left(1-p_{t}\right)\right]$ is a validation plot. Let $\left(\eta_{1}, \eta_{2}, \ldots, \eta_{n}\right)$ be a random sample from a Converse $\operatorname{GEV}(\gamma, \mu, \sigma)$, and let $\left(\eta_{(1)}, \eta_{(2)}, \ldots, \eta_{(n)}\right)$, be its order statistics. Let $p_{t}=\frac{t-0.5}{n}, t=1,2, \ldots, n$. If we plot $Y_{t}=-\log \left[1+\frac{\hat{\sigma}}{\hat{\gamma}}\left(\hat{\mu}-\eta_{(t)}\right)\right]$ against $X_{t}=\hat{\gamma} \log \left[-\log \left(1-p_{t}\right)\right]$ where $\hat{\gamma}, \hat{\mu}, \hat{\sigma}$ are the maximum likelihood estimates, $\hat{\eta}_{t}$ is the estimate of $\eta_{t}$, and the observations $\left(Y_{t}, X_{t}\right)$ fall on a straight line passing through the origin. This confirms that our assumption about the distribution is appropriate. We give a plot for our data in figure 7 . There is one outlier which corresponds to the minimum temperature in May 1958 (this was recorded as $-21.613^{\circ} \mathrm{C}$ ).

Consider the model (5.3) and we have (assuming the parameters are known)

$$
E\left(y_{t}\right)=\beta t+A \cos (\omega t)+B \sin (\omega t)+\frac{1}{1+\phi_{1}}\left[\mu+\frac{\sigma}{\gamma}-\frac{\sigma}{\gamma} \Gamma(1-\gamma)\right]
$$

Let $\hat{y}_{t}$ be the estimate of $E\left(y_{t}\right)$ when the parameters are replaced by their maximum likelihood estimates. By replacing $\hat{y}_{t}$ for $t=1,2, \ldots, 1000$, we observe that the estimated (expected) minimum temperatures will be above $0^{\circ} \mathrm{C}$ during the summer months indicating that there will be a longer melt season and this is suspected to be a primary contributing factor to major ice shelf break-ups (web: NASA, (2002)).

\section{(c) Prediction of the Minimum Temperatures}

Consider the time series $\left\{y_{t}\right\}$, where $y_{t}$ satisfies the model

$$
\begin{aligned}
y_{t} & =\beta_{1} t+A \cos (\omega t)+B \sin (\omega t)+e_{t} \\
e_{t}+\phi_{1} e_{t-1} & =\eta_{t} ; \eta_{t} \sim \operatorname{conv} \operatorname{GEV}(\gamma, \mu, \sigma)
\end{aligned}
$$




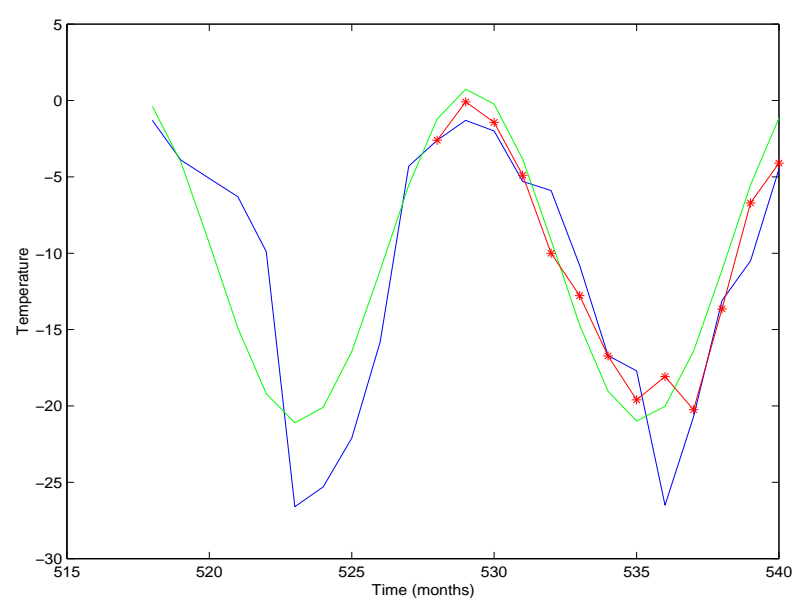

Figure 8. One step ahead forecasts of $\left\{y_{t}\right\}$ from $t=528$

Suppose we have observations $\left\{y_{s}, s \leq t_{0}\right\}$, then the minimum mean square error forecast of $y_{t_{0}+l}(l=1,2, \ldots)$ is given by

$$
\begin{aligned}
y_{t_{0}}(l) & =E\left(y_{t_{0}+l} \mid y_{s}, s \leq t_{0}\right) \\
& =\beta_{1}\left(t_{0}+l\right)+A \cos \left(\omega\left(t_{0}+l\right)\right)+B \sin \left(\omega\left(t_{0}+l\right)\right)+e_{t_{0}}(l) ; \\
e_{t_{0}}(l) & =-\phi_{1} e_{t_{0}}(l-1)+E\left(\eta_{t}\right) \\
E\left(\eta_{t}\right) & =\mu+\frac{\sigma}{\gamma} \Gamma(1-\gamma) .
\end{aligned}
$$

When $l=1$,

$$
\left[E\left(y_{t_{0}+1}-y_{t_{0}}(1)\right)\right]^{2}=\sigma_{\eta}^{2} .
$$

Usually we replace the parameters with their least squares estimates. For our forecasting purposes, we used the data given by the first 528 observations, and calculated the forecasts for the next twelve observations. The refitted model using the 528 observations is given by

$$
\begin{aligned}
y_{t}= & 0.0091036 t+10.652 \cos (0.52287 t)+2.45 \sin (0.52287 t)+e_{t} ; \\
& e_{t}-0.59228 e_{t-1}=\eta_{t} ; \\
\eta_{t} \sim & \operatorname{conv} \operatorname{GEV}(-0.1248,-4.3573,3.7454)
\end{aligned}
$$

The mean square error of the one step ahead forecast over the next 12 data points found to be 9.358 .

The plots of the data and the forecasts obtained from the model are given in figure 8 . Both the fit and the forecasts seem to indicate that the model fitted is adequate for the minimum temperatures.

\section{Model for the Maximum Temperatures}

As pointed out earlier, the range of the series is $\left(-8^{\circ} \mathrm{C}, 10^{\circ} \mathrm{C}\right)$, which is quite small compared to the minimum monthly temperatures. From the plot of the series, we 
cannot see any significant trend in the series. There is a yearly cycle, and from the boxplot (figure 5) we see that there are extremes in the summer months (January, February and March) and in the winter months (June, July and August). The extremes in summer months may cause melting of the ice shelves. In view of these observations we do not include a trend component in the model. Since we are analysing the maxima, it is natural for us to assume that the innovations have a Generalised Extreme Value distribution, $\operatorname{GEV}(\gamma, \mu, \sigma)$. We use the same procedure for estimating the model as before. The time series model for the maximum temperature is found to be

$$
\begin{aligned}
& y_{t}=1.6218 \cos (0.52337 t)+0.42645 \sin (0.52337 t)+e_{t} \\
& e_{t} \sim \operatorname{GEV}(-0.26568,1.7449,1.7199)
\end{aligned}
$$

It is interesting to note that the errors $\left\{e_{t}\right\}$ are mutually independent, but nonGaussian. This is in contrast to the minimum temperature data, which has correlated errors and has a linear trend and implies that the diurnal temperature range is decreasing and that asymmetric climate change is occurring in the Antarctic Peninsula.

Since the minimum temperatures are increasing and the maximum temperatures are remaining constant over the same period we believe the changes in minimum temperatures are more significant. In the following section we investigate this data further and see whether there is any relationship to ozone levels.

\section{Effect of Ozone Levels on Minimum Monthly Temperatures}

The influence of the human race on climate is still a matter for study and speculation, but the ability to perturb the ozone layer is an established fact. In this paper we aim to see if the amount of ozone in the stratosphere in the Antarctic Peninsula has a direct relationship to the minimum temperatures. If this can be established then it can be deduced that human activity does play some role in increasing the temperatures in the Antarctic Peninsula and future temperatures can be predicted with more certainty. Mean monthly ozone levels at the Faraday station are available from September 1957 to February 2002 (web: BASb, (2002)). These stratospheric ozone concentrations are recorded in Dobson units (DU) using a Dobson ozone spectrophotometer. This instrument tells us how much ozone there is in the atmosphere by comparing the intensities of two wavelengths of ultra-violet light from the sun. It is, therefore, not possible to make regular measurements of ozone during the Antarctic winter because the station is in darkness. Consequently, readings are missing for May, June and July each year. Readings are also missing for August 1958, 1959, 1969, 1970 and 1971 and April 1967. Therefore, there are 396 data points in this series spread over 534 months. We denote the ozone levels by $\left\{x_{t}\right\}$.

Missing values in August 1958, 1959, 1969, 1970 and 1971 are substituted with the August average over the 44 years, that is, 284 and April 1967 is substituted with the April average over the 44 year period, that is, 294. May, June and July for each year are substituted with the yearly average of that year, because there is significant random variation within each year. The series thus obtained, after 


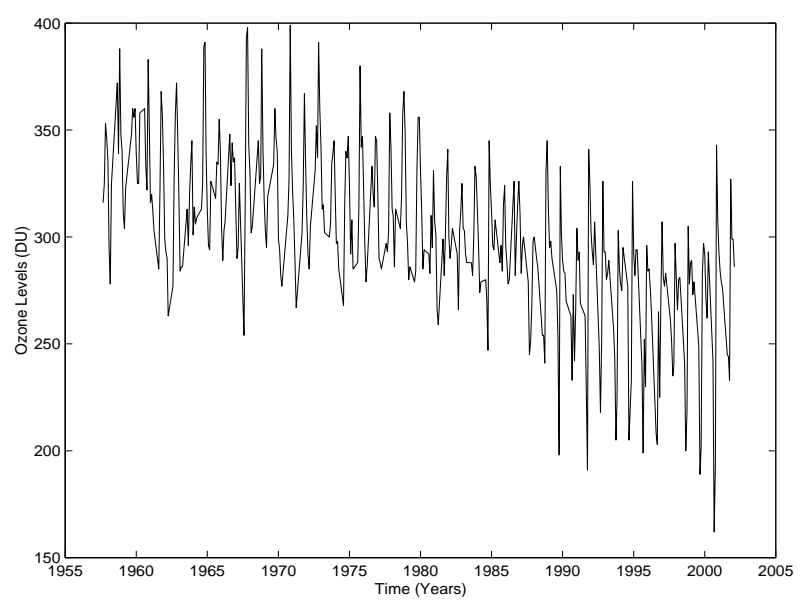

Figure 9. Ozone levels at the Faraday station (1957-2001)

substituting missing values, is denoted by $\left\{x_{t}^{\prime}, t=1,2, \ldots, 534\right\}$. Our object here is to find whether there is any significant relationship between ozone levels and the temperatures. In order to achieve this goal, we detrended and deseasonalised the series and then examined the cross correlation between the detrended and deseasonalised series. From the plots of the minimum temperatures (figure 1) and the ozone levels (figure 9), we see that there is a decrease in ozone levels during the years 1989 to 2001 and during the same period there is a steady increase in the minimum temperatures.

Using a similar methodology as described in previous sections for estimating the frequencies we observed that there are five dominant periods corresponding to 12 months, 11.7 months, 6 months, 4 months and 36 years and 9 months. The period corresponding to 12 months is the most dominant in the series as in minimum temperatures.

The model which fits best for the ozone levels is of the form

$$
x_{t}^{\prime}=341.39-0.14967 t+B_{t}+e_{t}
$$

where $\left\{e_{t}\right\}$ are independent random variables each distributed as normal with mean zero and standard deviation 17.50. Given that these observations are mean levels, the assumption of normality seems to be reasonable and is confirmed by standard tests. The seasonal component $B_{t}$ is given by

$$
\begin{aligned}
B_{t}= & 16.462 \cos (0.51805 t)+0.019499 \sin (0.51805 t) \\
& -10.077 \cos (1.0444 t)+15.541 \sin (1.0444 t) \\
& -8.0281 \cos (0.01425 t)-2.5313 \sin (0.01425 t) \\
& -2.6676 \cos (1.5664 t)+6.1399 \sin (1.5664 t) \\
& -4.3673 \cos (0.53481 t)+7.2745 \sin (0.53481 t)
\end{aligned}
$$

Since the data on ozone levels are available from September 1957 onwards, to standardise the two data sets (for differencing purposes) we considered new shifted 
series:

$$
\begin{aligned}
& x_{t}^{(2)}=\hat{x}_{t}^{\prime}+0.14967(t+1) \\
& y_{t}^{(2)}=y_{t}^{\prime}-0.008388(t+81)
\end{aligned}
$$

where $\left\{\hat{x}_{t}^{\prime}\right\}$ is the series obtained from $\left\{x_{t}^{\prime}\right\}$ given by (7.1) after deleting the intercept and the cyclical component $B_{t}$, and similarly $y_{t}^{\prime}$ is the series obtained from $y_{t}$ after deleting the trend and seasonal components. The sample correlation between $x_{t}^{(2)}$ and $y_{t}^{(2)}, \rho_{x_{t}^{(2)} y_{t}^{(2)}}(s),(s=0,1,2, \ldots)$ have been computed. The absolute maximum occurred at lag $s=1$ and the value is -0.129 . The corresponding $\mathrm{Z}$ statistic is

$$
Z=\left|\hat{\rho}_{x_{t}^{(2)} y_{t}^{(2)}}(1)\right| \sqrt{\frac{(N-2)}{1-\hat{\rho}_{x_{t}^{(2)} y_{t}^{(2)}}^{2}(1)}}=2.384
$$

where $N=339$ which suggests that there is a significant negative correlation at this lag. This indicates that higher ozone levels decrease the minimum temperatures and therefore prevent warming. The ozone is destroyed by gases such as $\mathrm{CO}_{2}$ and CFC's and reducing these means the ozone levels remain high and the minimum temperatures low. It can also be seen that there is a positive correlation between temperatures and time when we regress only on time. This indicates that the temperature has increased over time but the reason for this is not clear. Ozone levels may be one factor in this jigsaw as we see below.

We now consider the regression of $y_{t}$ on $x_{t-1}$ for the period from October 1957 to December 1995 (giving us 459 observations).

$$
y_{t}=\alpha x_{t-1}+\beta t+e_{t}
$$

where $\left\{e_{t}\right\}$ satisfies the $\operatorname{ARMA}(p, q)$ model

$$
\phi_{p}(B) e_{t}=\theta_{q}(B) \eta_{t} ; \quad \eta_{t} \sim \operatorname{conv} \operatorname{GEV}\left(\gamma, \mu, \sigma^{2}\right)
$$

In the model (7.3), the series $y_{t}$ and $x_{t}$ are deseasonalised only. The best model is found to be

$$
\begin{aligned}
& y_{t}=\underset{(0.0087,0.0025)}{-0.0156520}\left(x_{t-1}^{\prime}-B_{(t-1)+1}\right)+10.621 \cos (0.52291(t+81)) \\
& +2.5115 \sin (0.52291(t+81))+e_{t} ; \\
& \text { where } e_{t}=\underset{(0.034,0.032)}{0.58152} e_{t-1}+\eta_{t} ; \quad t=1,2, \ldots, 459
\end{aligned}
$$

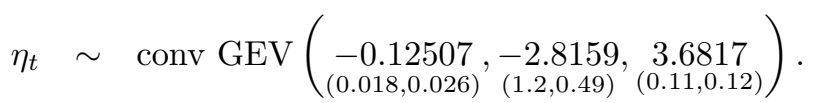

We have added 1 on to the time component in the periodic terms in $B_{t-1}$ and 81 on to the seasonal component corresponding to the minimum monthly temperatures. This is required because the sinusoids were estimated starting at $t=1$ (September 1957 for the ozone data and January 1951 for the temperature data). From the model (7.5), we see that high ozone levels lead to lower minimum temperatures and so decreasing the greenhouse gases should reduce the minimum monthly temperatures. 
From the above model, we can conclude that the minimum monthly temperatures appear to be increasing over time, but perhaps not as dramatically as was first thought. This increase in temperature appears to be determined only by the amount of ozone in the atmosphere since the time trend coefficient is not found to be significant. This indicates that increasing temperatures in the Antarctic Peninsula are caused by human activity and not by a natural phenomena.

\section{Acknowledgement}

We would like to thank the Engineering, Physical Sciences Research Council (U.K.) for supporting the research of Gillian L. Hughes.

\section{Appendix A. Extreme Value Distributions}

Suppose we have a sequence of independent, identically distributed random variables with cumulative distribution function $F(x)$. Let $M_{n}=\max \left(X_{1}, X_{2}, \ldots X_{n}\right)$. Then

$$
\operatorname{Pr}\left\{M_{n} \leq x\right\}=F^{n}(x)
$$

and for constants $a_{n}>0$, and $b_{n}$ so that as $n \longrightarrow \infty$

$$
\operatorname{Pr}\left\{\frac{\left(M_{n}-b_{n}\right)}{a_{n}} \leq x\right\}=F^{n}\left(a_{n} x+b_{n}\right) \rightarrow H(x) .
$$

When $H(x)$ is of the form

$$
H\left(x ; \mu_{X}, \sigma, \gamma\right)=\exp \left\{-\left[1+\gamma\left(\frac{x-\mu_{X}}{\sigma}\right)\right]^{-\frac{1}{\gamma}}\right\}, 1+\gamma\left(\frac{x-\mu_{X}}{\sigma}\right)>0
$$

we say the distribution is an Generalised Extreme Value (GEV) distribution. Here $\mu$ is a location parameter, $\sigma>0$ is a scale parameter and $\gamma$ is the shape parameter. The above distribution is very often used in modelling data from various disciplines (see for example, Smith (2001). The three special cases of $H(x)$ are Gumbel, Frechet and Weibull which are obtained by choosing the parameter $\gamma$ appropriately. The maximum likelihood estimation of these parameters $(\mu, \gamma, \sigma)$ was considered by Prescott and Walden (1980).

The expected value and variance of the random variable $X$ with the above distribution are given by

$$
\begin{aligned}
E(X) & =\left\{\begin{array}{c}
\xi+\frac{\sigma}{\gamma} \Gamma(1-\gamma) ; \gamma<1 \\
\mu+\varrho \sigma ; \gamma=0
\end{array}\right. \\
\operatorname{Var}(X) & =\left\{\begin{array}{c}
\frac{\sigma^{2}}{\gamma^{2}}\left[\Gamma(1-2 \gamma)-\Gamma^{2}(1-\gamma)\right] ; \gamma<\frac{1}{2} \\
\frac{\sigma^{2} \pi^{2}}{6} ; \gamma=0
\end{array}\right.
\end{aligned}
$$

(Laycock et al, (1990)) where $\xi=\mu-\frac{\sigma}{\gamma}$ and $\varrho$ is the Euler's constant.

We are often interested in the probabiliy distribution of $\operatorname{Min}\left(-x_{1},-x_{2}, \ldots,-x_{n}\right)=$ $-\operatorname{Max}\left(x_{1}, x_{2}, \ldots, x_{n}\right)$ which is given by

$$
F_{Y}(y)=1-\exp \left\{-\left[1+\gamma\left(\frac{-y+\mu_{Y}}{\sigma}\right)\right]^{-\frac{1}{\gamma}}\right\} ; 1+\gamma\left(\frac{-y+\mu_{Y}}{\sigma}\right)>0
$$


where $\mu_{y}=-E(X)$, and the corresponding probability density function is given by

$$
\begin{aligned}
f_{Y}(y)= & \frac{1}{\sigma} \exp \left\{-\left[1+\gamma\left(\frac{-y+\mu_{Y}}{\sigma}\right)\right]^{-\frac{1}{\gamma}}\right\}\left[1+\gamma\left(\frac{-y+\mu_{Y}}{\sigma}\right)\right]^{-\left(1+\frac{1}{\gamma}\right)} \\
& 1+\gamma\left(\frac{-y+\mu_{Y}}{\sigma}\right)>0
\end{aligned}
$$

The distribution (A 3) is known as the converse Generalised Extreme Value distribution, Conv $\operatorname{GEV}(\gamma, \mu, \sigma)$. The mean of $Y$ is given by

$$
E(Y)=\xi-\frac{\sigma}{\gamma} \Gamma(1-\gamma)
$$

and the variance of $Y$ is given by (A 2).

\section{Appendix B. Information Matrix}

We need the following expectations for evaluating the elements of the information matrix. Let

$$
v_{t}=1+\gamma\left(\frac{\mu-\eta_{t}}{\sigma}\right)
$$

where the random variable $\eta_{t}$ has a converse GEV distribution with parameters $(\gamma, \mu, \sigma)$. We can show (see Hughes (2002))

$$
\begin{aligned}
E\left[v_{t}^{k}\right] & =\Gamma(1-k \gamma) \\
E\left[v_{t}^{k} \log v_{t}\right] & =-\gamma \Gamma(1-k \gamma) \psi(1-k \gamma)
\end{aligned}
$$

where $\psi$ is the digamma function given by

$$
\psi(z)=\frac{\Gamma^{\prime}(z)}{\Gamma(z)}
$$

and

$$
\begin{aligned}
\psi(1) & =-\varrho \\
\psi(n) & =-\varrho+\sum_{j=1}^{n-1} j^{-1} ; n \geq 2, n \text { integer } \\
\psi(2+z) & =\frac{1}{1+z}+\psi(1+z) \\
\psi(1+z) & =-\varrho+\sum_{j=1}^{\infty} \frac{z}{j(j+z)} ; z \neq-1,-2,-3, \ldots
\end{aligned}
$$

Article submitted to Royal Society 
where $\varrho$ denotes Euler's constant. Furthermore,

$$
\begin{aligned}
E\left[\log v_{t}\right] & =\gamma \varrho \\
E\left[v_{t}^{-1} \log v_{t}\right] & =-\gamma \Gamma(1+\gamma)\left[-\varrho+\sum_{j=1}^{\infty} \frac{\gamma}{j(j+\gamma)}\right] \\
E\left[v_{t}^{-1 / \gamma} \log v_{t}\right] & =\gamma \Gamma(2)(\varrho-1) \\
E\left[v_{t}^{-(1+1 / \gamma)} \log v_{t}\right] & =-\gamma \Gamma(2+\gamma)\left[\frac{1}{1+\gamma}-\varrho+\sum_{j=1}^{\infty} \frac{\gamma}{j(j+\gamma)}\right] \\
E\left[v_{t}^{-(2+1 / \gamma)} \log v_{t}\right] & =-\gamma \Gamma(2+2 \gamma)\left[\frac{1}{1+2 \gamma}-\varrho+\sum_{j=1}^{\infty} \frac{2 \gamma}{j(j+2 \gamma)}\right] \\
E\left[v_{t}^{k} \log v_{t}\left(\mu-\eta_{t}\right)\right] & =\sigma \Gamma(1-k \gamma) \psi(1-k \gamma)-\sigma \Gamma(1-\gamma(k+1)) \psi(1-\gamma(k+1)) \\
E\left[v_{t}^{-(1+1 / \gamma)} \log v_{t}\left(\mu-\eta_{t}\right)\right] & =\sigma \Gamma(2+\gamma) \psi(2+\gamma)+\sigma \varrho-\sigma \\
E\left[v_{t}^{k}\left(\log v_{t}\right)^{2}\right] & =\gamma^{2} \Gamma^{\prime \prime}(1-k \gamma) \\
E\left[v_{t}^{-1 / \gamma}\left(\log v_{t}\right)^{2}\right] & =\gamma^{2}\left[\frac{\pi^{2}}{6}+\varrho^{2}-2 \varrho\right] \\
E\left[v_{t}^{k}\left(\mu-\eta_{t}\right)\right] & =-\frac{\sigma}{\gamma} \Gamma(1-k \gamma)+\frac{\sigma}{\gamma} \Gamma(1-\gamma(k+1)) \\
E\left[\eta_{t}^{2}\right] & =\left(\mu+\frac{\sigma}{\gamma}\right)^{2}-2 \frac{\sigma}{\gamma}\left(\mu+\frac{\sigma}{\gamma}\right) \Gamma(1-\gamma)+\frac{\sigma^{2}}{\gamma^{2}} \Gamma(1-2 \gamma)
\end{aligned}
$$

We can use these expressions to obtain the elements of Fisher's Information Matrix. The second derivatives of $\eta_{t}$ are given below

$$
\begin{aligned}
E\left[\frac{\partial \eta_{t}}{\partial \beta_{l}} \frac{\partial \eta_{t}}{\partial \beta_{k}}\right] & =\left[\theta^{-1}(B) \phi(B) x_{l t}\right]\left[\theta^{-1}(B) \phi(B) x_{k t}\right] \\
E\left[\frac{\partial \eta_{t}}{\partial \phi_{l}} \frac{\partial \eta_{t}}{\partial \phi_{k}}\right] & =\sum_{j=0}^{\infty} \varphi_{j} \varphi_{|k-l|+j} \operatorname{Var}\left(\eta_{t}\right)+\left(\sum_{j=0}^{\infty} \varphi_{j}\right)^{2} E\left[\eta_{t}\right]^{2} \\
E\left[\frac{\partial \eta_{t}}{\partial \theta_{l}} \frac{\partial \eta_{t}}{\partial \theta_{k}}\right] & =\sum_{j=0}^{\infty} \tau_{j} \tau_{|k-l|+j} \operatorname{Var}\left(\eta_{t}\right)+\left(\sum_{j=0}^{\infty} \tau_{j}\right)^{2} E\left(\eta_{t}\right)^{2} \\
E\left[\frac{\partial \eta_{t}}{\partial \phi_{l}} \frac{\partial \eta_{t}}{\partial \beta_{k}}\right] & =-\theta^{-1}(B) \phi(B) x_{k t} \phi^{-1}(1) E\left[\eta_{t}\right] \\
E\left[\frac{\partial \eta_{t}}{\partial \theta_{l}} \frac{\partial \eta_{t}}{\partial \beta_{k}}\right] & =\theta^{-1}(B) \phi(B) x_{k t} \theta^{-1}(1) E\left[\eta_{t}\right] \\
E\left[\frac{\partial \eta_{t}}{\partial \theta_{l}} \frac{\partial \eta_{t}}{\partial \phi_{k}}\right] & =-\sum_{j=0}^{\infty} \tau_{j} \varphi_{|k-l|+j} \operatorname{Var}\left(\eta_{t}\right)-\sum_{j=0}^{\infty} \tau_{j} \sum_{m=0}^{\infty} \varphi_{m} E\left[\eta_{t}\right]^{2}
\end{aligned}
$$


and the expected value of the first derivatives are

$$
\begin{aligned}
E\left[\frac{\partial \eta_{t}}{\partial \beta_{k}}\right] & =-\theta^{-1}(B) \phi(B) x_{k t} \quad(k=1, \ldots, r) \\
E\left[\frac{\partial \eta_{t}}{\partial \phi_{k}}\right] & =\phi^{-1}(1) E\left(\eta_{t}\right) \quad(k=1, \ldots, p) \\
E\left[\frac{\partial \eta_{t}}{\partial \theta_{k}}\right] & =-\theta^{-1}(1) E\left(\eta_{t}\right) \quad(k=1, \ldots, q)
\end{aligned}
$$

and the expected values of the second derivatives are

$$
\begin{aligned}
E\left[\frac{\partial^{2} \eta_{t}}{\partial \phi_{l} \partial \beta_{k}}\right] & =-\theta^{-1}(B) x_{k, t-l} \\
E\left[\frac{\partial^{2} \eta_{t}}{\partial \theta_{l} \partial \beta_{k}}\right] & =-\theta^{-2}(B) \phi(B) x_{k t} \\
E\left[\frac{\partial^{2} \eta_{t}}{\partial \theta_{l} \partial \phi_{k}}\right] & =-\theta^{-1}(1) \phi^{-1}(1) E\left(\eta_{t}\right) \\
E\left[\frac{\partial^{2} \eta_{t}}{\partial \theta_{l} \partial \theta_{k}}\right] & =2 \theta^{-2}(1) E\left(\eta_{t}\right) .
\end{aligned}
$$

and

$$
\begin{aligned}
E\left[\frac{\partial^{2} l}{\partial \beta_{l} \partial \beta_{k}}\right]= & \frac{-(1+\gamma)^{2}}{\sigma^{2}} \Gamma(1+2 \gamma) \sum_{t=s+1}^{n}\left[\theta^{-1}(B) \phi(B) x_{l t}\right]\left[\theta^{-1}(B) \phi(B) x_{k t}\right] \\
E\left[\frac{\partial^{2} l}{\partial \phi_{l} \partial \beta_{k}}\right]= & \frac{-(1+\gamma)^{2}}{\sigma^{2}} \Gamma(1+2 \gamma) \sum_{t=s+1}^{n}\left(-\theta^{-1}(B) \phi(B) x_{k t} \phi^{-1}(1) E\left[\eta_{t}\right]\right) \\
E\left[\frac{\partial^{2} l}{\partial \theta_{l} \partial \beta_{k}}\right]= & \frac{-(1+\gamma)^{2}}{\sigma^{2}} \Gamma(1+2 \gamma) \sum_{t=s+1}^{n} \theta^{-1}(B) \phi(B) x_{k t} \theta^{-1}(1) E\left[\eta_{t}\right] \\
E\left[\frac{\partial^{2} l}{\partial \phi_{l} \partial \phi_{k}}\right]= & \frac{-(n-s)(1+\gamma)^{2}}{\sigma^{2}} \Gamma(1+2 \gamma)\left[\sum_{j=0}^{\infty} \varphi_{j} \varphi_{|k-l|+j} \operatorname{Var}\left(\eta_{t}\right)+\left(\sum_{j=0}^{\infty} \varphi_{j}\right)^{2} E\left(\eta_{t}\right)^{2}\right] \\
E\left[\frac{\partial^{2} l}{\partial \theta_{l} \partial \phi_{k}}\right]= & \frac{(n-s)(1+\gamma)^{2}}{\sigma^{2}} \Gamma(1+2 \gamma)\left[\sum_{j=0}^{\infty} \tau_{j} \varphi_{|k-l|+j} \operatorname{Var}\left(\eta_{t}\right)+\sum_{j=0}^{\infty} \tau_{j} \sum_{m=0}^{\infty} \varphi_{m} E\left(\eta_{t}\right)^{2}\right] \\
E\left[\frac{\partial^{2} l}{\partial \theta_{l} \partial \theta_{k}}\right]= & \frac{-(n-s)(1+\gamma)^{2}}{\sigma^{2}} \Gamma(1+2 \gamma)\left[\sum_{j=0}^{\infty} \tau_{j} \tau_{|k-l|+j} \operatorname{Var}\left(\eta_{t}\right)+\left(\sum_{j=0}^{\infty} \tau_{j}\right)^{2} E\left(\eta_{t}\right)^{2}\right] \\
E\left[\frac{\partial^{2} l}{\partial \beta_{k} \partial \gamma}\right]= & \frac{\left(\gamma^{2}+\gamma+1\right)}{\sigma \gamma^{2}} \Gamma(1+\gamma) \sum_{t=s+1}^{n} E\left[\frac{\partial \eta_{t}}{\partial \beta_{k}}\right]-\frac{(1+\gamma)^{2}}{\sigma \gamma^{2}} \Gamma(1+2 \gamma) \sum_{t=s+1}^{n} E\left[\frac{\partial \eta_{t}}{\partial \beta_{k}}\right] \\
& +\frac{1}{\gamma \sigma} \Gamma(2+\gamma) \psi(2+\gamma) \sum_{t=s+1}^{n} E\left[\frac{\partial \eta_{t}}{\partial \beta_{k}}\right]
\end{aligned}
$$

Article submitted to Royal Society 


$$
\begin{aligned}
& \frac{1}{n-s} E\left[\frac{\partial^{2} l}{\partial \phi_{k} \partial \gamma}\right]=\frac{\left(\gamma^{2}+\gamma+1\right)}{\sigma \gamma^{2}} \Gamma(1+\gamma) E\left[\frac{\partial \eta_{t}}{\partial \phi_{k}}\right]-\frac{(1+\gamma)^{2}}{\sigma \gamma^{2}} \Gamma(1+2 \gamma) E\left[\frac{\partial \eta_{t}}{\partial \phi_{k}}\right] \\
& +\frac{1}{\gamma \sigma} \Gamma(2+\gamma) \psi(2+\gamma) E\left[\frac{\partial \eta_{t}}{\partial \phi_{k}}\right] \\
& \frac{1}{n-s} E\left[\frac{\partial^{2} l}{\partial \theta_{k} \partial \gamma}\right]=\frac{\left(\gamma^{2}+\gamma+1\right)}{\sigma \gamma^{2}} \Gamma(1+\gamma) E\left[\frac{\partial \eta_{t}}{\partial \theta_{k}}\right]-\frac{(1+\gamma)^{2}}{\sigma \gamma^{2}} \Gamma(1+2 \gamma) E\left[\frac{\partial \eta_{t}}{\partial \theta_{k}}\right] \\
& +\frac{1}{\gamma \sigma} \Gamma(2+\gamma) \psi(2+\gamma) E\left[\frac{\partial \eta_{t}}{\partial \theta_{k}}\right] \\
& \frac{1}{n-s} E\left[\frac{\partial^{2} l}{\partial \mu \partial \gamma}\right]=-\frac{\left(\gamma^{2}+\gamma+1\right)}{\sigma \gamma^{2}} \Gamma(1+\gamma)+\frac{(1+\gamma)^{2}}{\sigma \gamma^{2}} \Gamma(1+2 \gamma) \\
& -\frac{1}{\gamma \sigma} \Gamma(2+\gamma) \psi(2+\gamma) \\
& \frac{1}{n-s} E\left[\frac{\partial^{2} l}{\partial \sigma \partial \gamma}\right]=-\frac{\left(\gamma^{2}+2 \gamma+2\right)}{\sigma \gamma^{3}} \Gamma(1+\gamma)+\left(\frac{1+\gamma}{\sigma \gamma^{3}}\right) \\
& +\frac{(1+\gamma)^{2}}{\sigma \gamma^{3}} \Gamma(1+2 \gamma)-\frac{1}{\gamma^{2} \sigma} \Gamma(2+\gamma) \psi(2+\gamma)-\frac{1}{\gamma^{2} \sigma} \varrho ; \\
& \frac{1}{n-s} E\left[\frac{\partial^{2} l}{\partial \gamma^{2}}\right]=\frac{-2}{\gamma^{2}} \varrho+\frac{2\left(\gamma^{2}+\gamma+1\right)}{\gamma^{4}} \Gamma(1+\gamma)+\frac{(\gamma+1)(\gamma-1)}{\gamma^{4}} \\
& -\frac{(\gamma+1)^{2}}{\gamma^{4}} \Gamma(1+2 \gamma)-\frac{2}{\gamma^{2}} \psi(2)-\frac{1}{\gamma^{2}}\left[\psi^{(1)}(2)+\psi(2)^{2}\right] \\
& +\frac{2}{\gamma^{3}}[\Gamma(2+\gamma) \psi(2+\gamma)-\psi(2)] \text {; } \\
& \frac{1}{n-s} E\left[\frac{\partial^{2} l}{\partial \mu^{2}}\right]=\frac{-(\gamma+1)^{2}}{\sigma^{2}} \Gamma(1+2 \gamma) ; \\
& \frac{1}{n-s} E\left[\frac{\partial^{2} l}{\partial \sigma^{2}}\right]=-\frac{1}{\sigma^{2} \gamma^{2}}+\frac{2(1+\gamma)}{\sigma^{2} \gamma^{2}} \Gamma(1+\gamma)-\frac{(1+\gamma)^{2}}{\sigma^{2} \gamma^{2}} \Gamma(1+2 \gamma) \\
& \frac{1}{n-s} E\left[\frac{\partial^{2} l}{\partial \mu \partial \sigma}\right]=-\frac{(1+\gamma)^{2}}{\sigma^{2} \gamma} \Gamma(1+2 \gamma)+\frac{(1+\gamma)}{\sigma^{2} \gamma} \Gamma(1+\gamma) . \\
& E\left[\frac{\partial^{2} l}{\partial \beta_{k} \partial \mu}\right]=\frac{(1+\gamma)^{2}}{\sigma^{2}} \Gamma(1+2 \gamma) \sum_{t=s+1}^{n} E\left[\frac{\partial \eta_{t}}{\partial \beta_{k}}\right] \\
& \frac{1}{n-s} E\left[\frac{\partial^{2} l}{\partial \phi_{k} \partial \mu}\right]=\frac{(1+\gamma)^{2}}{\sigma^{2}} \Gamma(1+2 \gamma) E\left[\frac{\partial \eta_{t}}{\partial \phi_{k}}\right] \\
& \frac{1}{n-s} E\left[\frac{\partial^{2} l}{\partial \theta_{k} \partial \mu}\right]=\frac{(\gamma+1)^{2}}{\sigma^{2}} \Gamma(1+2 \gamma) E\left[\frac{\partial \eta_{t}}{\partial \theta_{k}}\right]
\end{aligned}
$$

Article submitted to Royal Society 


$$
\begin{aligned}
E\left[\frac{\partial^{2} l}{\partial \beta_{k} \partial \sigma}\right]= & \frac{(1+\gamma)^{2}}{\sigma^{2} \gamma} \Gamma(1+2 \gamma) \sum_{t=s+1}^{n} E\left[\frac{\partial \eta_{t}}{\partial \beta_{k}}\right] \\
& -\frac{(1+\gamma)}{\sigma^{2} \gamma} \Gamma(1+\gamma) \sum_{t=s+1}^{n} E\left[\frac{\partial \eta_{t}}{\partial \beta_{k}}\right] \\
\frac{1}{n-s} E\left[\frac{\partial^{2} l}{\partial \phi_{k} \partial \sigma}\right]= & \frac{(1+\gamma)^{2}}{\sigma^{2} \gamma} \Gamma(1+2 \gamma) E\left[\frac{\partial \eta_{t}}{\partial \phi_{k}}\right] \\
\frac{(1+\gamma)}{\sigma^{2} \gamma} \Gamma(1+\gamma) E\left[\frac{\partial \eta_{t}}{\partial \phi_{k}}\right] & -\frac{(1+\gamma)^{2}}{\sigma^{2} \gamma} \Gamma(1+2 \gamma) E\left[\frac{\partial \eta_{t}}{\partial \theta_{k}}\right] \\
\left.=\frac{\partial^{2} l}{\partial \theta_{k} \partial \sigma}\right]= & -\frac{(1+\gamma)}{\sigma^{2} \gamma} \Gamma(1+\gamma) E\left[\frac{\partial \eta_{t}}{\partial \theta_{k}}\right] .
\end{aligned}
$$

\section{References}

BAS(a), 2002 British Antarctic Survey Report,

http://www . antarctica.ac.uk/AboutAntarctica/Weather/Temperature/Temperature.html.

BAS(b), 2002 British Antarctic Survey Report,

http://www .antarctica.ac.uk/met/jds/ozone/data/foz5699.dat

Brockwell And Davies, 1996, "Time Series: Theory and Methods", Springer, New York.

http://www . antarctica.ac.uk/met/jds/ozone/data/foz5699.dat.

Budd, W.F., Jenson, D., Mavrakis, E. And Coutts. B, 1994, "Modelling the Antarctic ice-sheet through time", Annals of Glaciology, Vol. 20, pp. 291-297.

D’Agostino, R.B. And Stephens, M.A., 1986, Goodness-of-fit Techniques, Marcel Dekker, Inc., New York.

Gumbel, E.J., 1958, Statistics of Extremes, Columbia University Press, New York.

Hannan, E.J. And Rissanen, J., 1982, "Recursive estimation of mixed autoregressivemoving average order", Biometrika, Vol. 69, pp. 81-94.

Hughes, G. L., 2002 "Multiple Regression with correlated, non Gaussian errors - An analysis of extreme temperature data. ", Phd thesis submitted to the University of Manchester Institute of Science and Technology, Manchester.

Hulbe, C.L., 1997, "Recent changes to Antarctic Peninsula ice shelves: what lessons have been learned?", Natural Science, Vol. 1., Article 6, http://naturalscience.com/ns/articles/01-06/ns_clh.html.

Huybrechts. P. And Oerlemans, J.,1990, "Response of the Antarctic ice sheet to future greenhouse warming", Climate Dynamics, Vol. 5, pp. 93-102.

Kavalieris, L. And Hannan, E.J., 1994, Kavalieris, L. and Hannan, E.J., 1994, "Determining the number of terms in a trigonometric regression", Journal of Time Series Analysis, Vol. 15, pp. 613-625.

Laycock, P.J., CotTis, R.A., Scarf, P.A.,1990, "Extrapolation of extreme pit depths in space and time", Journal of the Electrochemical Society, Vol. 137, pp. 64-69. 
NASA, 2002, NASA/Report, http://earthobservatory.nasagov/newsroom/nasanews/2001/20010164406.html.

Prescott, P. And Walden, A.T., 1980, "Maximum likelihood estimation of the parameters of the generalized extreme-value distribution",Biometrika, Vol. 67, pp. 723-724.

Quinn, B.G. And Fernandes, J.M., 1991, "A fast and efficient technique for the estimation of frequency", Biometrika, Vol. 78, pp.489-498.

Reiss, R. D. And Thomas, M. , 1997, Statistical Analysis of Extreme Values, Birkhauser, Basel.

Sмiтh, R. L. 2001 "Extreme value statistics in meterology and environment part 1 ", Notes from CBMS course in Environmental statistics, University of Washigton.

http://www.stat.unic.edu/postcript/rs/envstat/env.html 